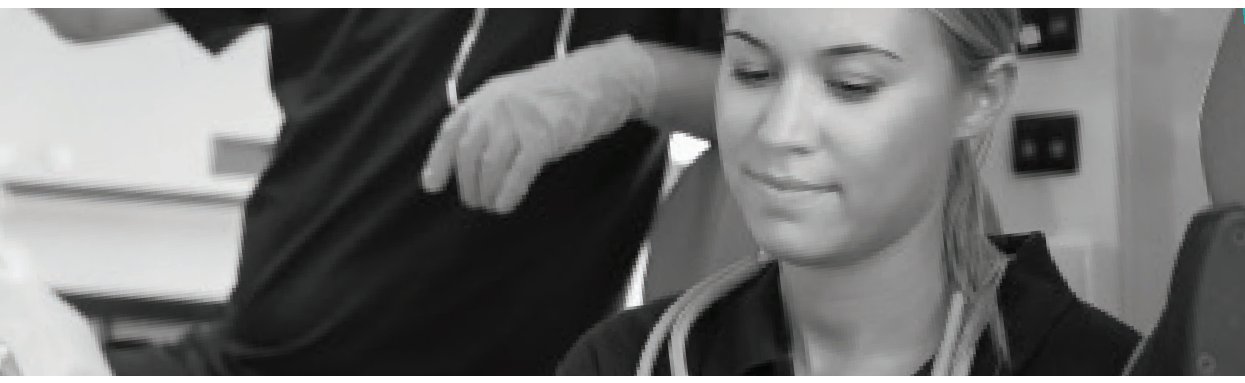

Volume 13 | Issue 3 | Article 1
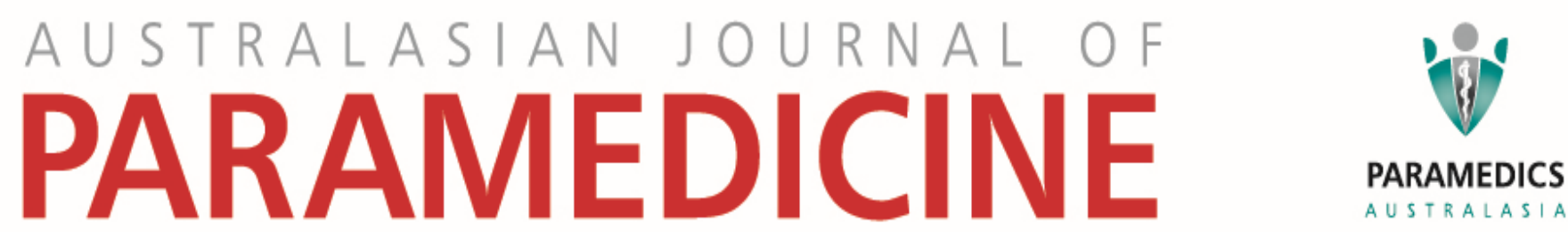

\title{
Paramedical ethics during acute emergency medical service referral
}

Anthony Campeau

Sunnybrook Centre for Pre-hospital Care, Ontario, Canada 


\title{
Paramedical ethics during acute emergency medical service referral
}

\author{
Anthony Campeau Ed.D, Paramedic Research Specialist ${ }^{1}$
}

Affiliation:

${ }^{1}$ Sunnybrook Centre for Pre-hospital Care, affiliated with the University of Toronto, Ontario, Canada

\section{Abstract}

\section{Introduction}

Ethical dilemmas comprise an important non-technical aspect of paramedicine but have not received significant research attention. This study explores the nature of paramedical ethics during the high-stakes referral of emergency ambulance patients, and relates findings to accepted concepts of professionalism.

\section{Methods}

Grounded Theory Methodology was used to analyse interview data from paramedics in Ontario, Canada, who had experience making field-referrals for patients suffering from ST elevation myocardial infarction and acute stroke.

Results

Paramedics describe extensive use of an ethic of applied beneficence, and patient advocacy during calls on a borderline for inclusion in referrals to medical specialists.

\section{Conclusion}

The management of complex ethical dilemmas is often cited as an example of professionalism. Paramedics have developed a unique type of ethicality to address the complexities of emergency field-referrals that reflects their field of practice, and is consistent with the literature on professional level ethical practice.

Keywords:

paramedic, ethics, professional, referral

Corresponding author: Anthony Campeau: anthony.campeau@sunnybrook.ca 


\section{Introduction}

Ethical dilemmas are widely accepted as a reality of practice in the health professions, and are often a consequence of the complexity of negotiating pragmatic decisions in situations that involve a range of personal and institutional values. This complexity is often compounded by uncertain clinical outcomes, and the ability to manage these situations is regularly cited as a hallmark of professionalism (1).

Paramedics are no strangers to such ethical dilemmas. They frequently encounter a number of ethical challenges, including patient situations involving advance care directives (do not resuscitate orders), involuntary transports, limitations on scopes of practice, complications with consent, and uncertainties about their duty to respond to emergencies occurring in unsafe areas such as active crime scenes.

While it is true that regulatory and occupational organisations provide some attention to ethical issues in the form of regulatory standards and codes of conduct $(2,3)$, their treatment in these areas is limited to general or high-level conceptual narratives that are operationalised as bestpractice statements. The general nature of these narratives does not provide direction on how to apply them in a specific context or offer guidance that is sufficient for making complex decisions in the field. This situation reflects an imbalance in the range of pre-hospital research. Specifically, research into the objective or technical aspects of paramedicine is robust. Articles on medical scopes of practice, procedural precision and correctness are common (and important), yet the same attention is not paid to paramedicine's non-technical (4) aspects: such as how paramedics deal with ethical dilemmas.

This imbalance is easily illustrated when we consider the case of paramedics performing field-based referrals of patients with two serious medical conditions: ST elevation myocardial infarction (STEMI), and acute stroke. An online multi-search using criteria describing emergency medical service STEMI (EMS-STEMI) referral programs, identified 578 articles between 1999 and 2014. The same search process using criteria describing EMS acute stroke referral programs identified 1924 articles. Most of this research focuses on the ability of paramedics to correctly diagnose patient conditions (5), or other technical topics such as clinical outcomes (6-8). None had the word 'ethics' in the title.

Understanding the ethics involved in paramedicine is an important research topic as it deals with the affective motivations (9) of paramedics. Inquiry in this area offers insights that can complement those obtained through inquiries into cognitive processes (10) and, taken together, provide a more complete understanding of paramedic decision-making. My use of ethics here is closely aligned with that of Purtilo and Doherty's (11) perspective that each health care profession has a form of ethics that develops out of its role. This study into paramedical ethics explores subjective concerns expressed by paramedics and interprets them using ethical concepts commonly found among the health professions. While there have been calls for increased attention in this area (12), actual research into paramedical ethics remains rare, leaving paramedics to draw only on their personal, rather than collective experiences.

In this qualitative paper I focus on this under-researched area by exploring how paramedics have developed a unique ethical approach to operationalising high-stakes referral, and relate this to selected aspects of professionalism.

\section{Background}

Paramedics in Ontario (Canada) now regularly perform fieldbased referral of selected patients to medical specialists. In these programs, medical specialists have identified clinical criteria intended to identify patients suffering from STEMI or an acute stroke. These patients have a high likelihood of benefitting from special, time sensitive in-hospital interventions $(5,6)$. Paramedics transport patients who meet the criteria directly from the scene to regional referral/treatment centres.

This is a major change in practice for paramedic and introduces two new influences on the ethical aspects of their decisionmaking in the field. First, paramedics must now make riskrelated judgements about patient tolerance for longer transport times to regional rather than local centres, and second, they have an unprecedented level of direct inter-professional practice with medical specialists.

Ethical dilemmas are in large part situational, and so it may be helpful to briefly describe the occupational nature of paramedic practice. Specifically, the working lives of paramedics are unique and the distinguishing feature is not the medical scope of practice per se, but rather where it is practised $(13,14)$. More specifically, as Nelson (15) has explained, paramedics practise 'in a context rife with chaotic, dangerous and often uncontrollable elements with which hospital based practitioners need not contend' (p. 162). Paramedics must develop the occupational knowledge to transfer medical knowledge learned in the classroom to non-medical environments, ie. to be able to practise 'in the street'. Although there has been limited research into the complexities of this process, in other work (16) I have described how paramedics must 'fit' medicine into their work context by controlling the social and physical space at an emergency scene, and furthermore that skill in this area is what paramedics believe comprises expertise in the occupation (17).

Paramedics must now fit field-referrals into their work context, mixing objective clinical criteria with the subjective nontechnical ethical aspects of individual cases. 


\section{Methods}

As there is little formal research into the ethical aspects of how paramedics implement EMS referral an exploratory approach was required. Additionally, EMS referral must be activated by paramedics on an individualised basis therefore it was important to obtain an understanding of practitioners' points of view. This necessitated an approach that would emphasise the context of referral, provide in-depth data and draw on highly personalised perspectives (18). The qualitative method of Grounded Theory Methodology (GTM) was therefore an appropriate choice. It is ideally suited for exploring social contexts and processes and for emphasising the participant's perspective (19). Grounded Theory Methodology utilises a systematic, inductive and cyclical data collection and analysis approach to discover hidden social processes that are subsequently organised and abstracted into concepts (20).

Interviews were selected as a source of data as they offer several features that are appropriate when exploring new areas of inquiry. These include allowing participants to reflect on and express their personal experiences and views on the research topic. They also allow the acquisition of in-depth information (21). However, an associated caveat is that these data describe what participants say rather that the performance data that would be provided by observational studies.

\section{Participants}

The participants in this study were all paramedic employees of a large sub-urban land ambulance service in Ontario (Canada) working in two person ambulance crews. The ambulance service operates several bases, all of which have catchment areas with the potential for transport to both local and regional referral centres. All paramedics had several years of experience with the EMS referral program. Their employer agreed to allow participation on a voluntary, confidential and anonymous (to anyone other than the researcher) basis, with the researcher contacting participants directly. All participants signed a voluntary informed consent agreement and were required to meet the following criteria:

1. Have current paramedic credentials as required by provincial regulations.

2. Be employed as a paramedic in a certified land ambulance service actively utilising EMS-STEMI and acute stroke referral programs.

3. Have received in-service training on the use of EMS-STEMI and acute stroke referral protocols.

4. Have personal experience using EMS-STEMl and acute stroke referral.

\section{Data collection and analysis}

Generic letters of invitation to participate in the study were distributed to the ambulance service's bases and posted in prominent areas for paramedics to read. These letters outlined the aim of the study and data collection format, and also confirmed that participation would be voluntary and confidential. Interviews were scheduled as paramedics responded to invitations to participate. Data were obtained using in-depth, private interviews. These interviews varied in length from 1 to 2 hours. Interviews were audiotaped and the recordings were subsequently transcribed to facilitate analysis. Consistent with GTM, data collection and analysis were conducted simultaneously, using constant comparison (19) to progressively develop a conceptual understanding of the social processes in play. Data were obtained during an initial round of five interviews and the GTM theoretical sampling technique (19) was used to identify information that was highly relevant to emerging concepts. As tentative concepts were identified, interview questions were revised and a second round of five interviews was conducted, using the same approach, for a total of 10 paramedics. Eight of the paramedics were men, and two were women. Cessation of sampling occurred once theoretical saturation (20) was reached, meaning that no new information was forthcoming.

\section{Ethics approval}

This human subject research was approved by Sunnybrook Health Sciences Centre, Research Ethics Board. Also, fictitious names have been used to describe real towns/cities and the confidentiality of paramedics' identity preserved by using artificial initials for attributing narratives.

\section{Results}

How paramedics describe the ethics of incorporating referrals into their practice can be organised into two main categories:

- an ethicality of applied beneficence

- patient advocacy during borderline cases.

\section{An ethicality of applied beneficence}

Paramedics' descriptions of their motivations to refer patients emphasises an ethicality of applied beneficence. Paramedics describe a strong level of empathy for the vulnerability of their patients and within their scene-management strategies, look for opportunities to act, in the judgement of paramedics, in their patients' best interests. This seems to create a motivation to give their patients access to referral procedures whenever possible. As explained by 'GH', 'these programs get the patients to the right care at the right time'. Similarly, 'LM' said, 'Getting them to the right hospital, the proper hospital helps them immensely. If there are people there that are higher trained [specialists] we need to get our patients there'. And again by 'AK', 'My motivation is to get the patient the best care available'. 
Conversely, paramedics describe frustration when they cannot act in the best interests of their patients. For example, 'LM' describes his reaction when patients are clearly not eligible for referral: 'You feel - it's tough you know. If I am up in Anytown 1 [a remote community] you know that if that same patient were in Anytown II [community close to a regional centre] I could just take them to the [regional centre]'. And further, 'EW' advises, 'It sounds kind of corny, but I always think, what if this were my dad - I would want to try'.

\section{Patient advocacy during borderline cases}

Paramedics are well aware of the objective inclusion/exclusion criteria for these programs, but these criteria can be considered decontextualised insofar as they assume precise knowledge is available. In fact, important information needed to meet criteria such as case history and time of events, needs to be acquired from a range of sources during high stress and often confused circumstances. This context introduces significant uncertainty to the quality of this information.

As explained by 'JP': 'For stroke if it's vague from the patient, they can't communicate, we go to the family, and then it becomes difficult. Then we work our way back with the family: well, when was the last time you saw them in a normal state? If we can establish that time, then we piece meal it and work forward. It's difficult for sure'.

The estimated nature of the event-times involved in these situations results in cases that are borderline for inclusion. In these circumstances paramedics appear respectful of the time criteria and describe considerable pressure to comply with it, yet also want to act in the best interests of their patient by advocating with receiving physicians. Paramedics are also extremely sensitive to the potential for these cases to cross the professional boundary represented by the criteria (if they were to transport a patient who was clearly not eligible) and so they only advocate for selected cases where there is close proximity to the criteria. As expressed by 'NP': 'If I can get a close enough time [to the eligibility criteria] I am calling the interventionist. If I have enough to convince the receiving doctor then I will use that. But if it's way outside the window [of eligibility] then we are for sure going to the closest hospital'.

So paramedics will advocate for selected patients. 'LM' explains, 'I've tried to convince the interventionist or the doctor - here are my reasons'. However, paramedics only use this real-time advocacy if the rest of the clinical picture demonstrates an otherwise eligible patient. As expressed by 'EW': 'I think it's a balance of pros and cons. If you take the whole picture of the patient, if you are positive they would benefit from the treatment, we make the call to see if we are allowed to do that [transport to regional centre]'.

'AK' elaborates: 'If it's right on the bubble, and that exact scenario has happened to me more than once. A big part is patient presentation, how much elevation is in the 12 lead, which leads, their vitals etc. if in my opinion at that time they need the help. I know the ideal treatment is at [the centre]'.

In these cases, paramedics are particularly attentive to their patient assessments as there is at least more certainty with respect to these physical measurements, which may help tip the eligibility balance. As stated by 'TB', 'If I am calling a specialist, I am getting all my ducks in a row for sure'.

\section{Augmented advocacy}

Paramedics also try to augment their advocacy by optimising those aspects of the call that are under their exclusive control. These actions can be considered a type of augmented advocacy, because they are under the paramedics' control, yet initiated to support their advocacy for referral. Stated in ethical terms, these actions can be understood as enhancing beneficence. Key among these is an effort to minimise the on-scene time. Paramedics know that on a borderline call, precious minutes lost at the scene may disqualify a patient and so they make operational changes to their routine in order to advance the patient's eligibility. These efforts can be organised into three areas: a) changing the division of labour and pace at the scene, b) delaying interventions until enroute, and c) identifying modifiable drive-time factors. Paramedics hope that by minimising their on-scene and drive times, they can provide a patient with access to referral treatments.

In terms of changing the division of labour, paramedics will improvise to meet the requirements for a consultation with a specialist. As explained by 'AK', 'I give more tasks to my partner [obtain patient's history], I still need the info as I can't patch [to a specialist] without the needed information. I will try to get information from more than one source'. In another example, 'KH' advises, 'If I find the time is an issue, then get them on the stretcher, and we will figure it out enroute. These are times to hustle'. And according to 'GH', 'I get my partner to pack up [assemble patient care equipment] fast'.

Similarly, in terms of changing pace, the delaying of treatments until later in the call is another method to minimise scene time. As 'GH' says, 'You move your butt. You do as much as you can in the driveway'. Sometimes, this strategy presents paramedics with dilemmas such as described by 'AK', 'We will forego treatments [on-scene] until we are mobile like starting an IV [intravenous], but that's its own dilemma if due to vital signs you may want that IV sooner rather than later'.

Finally, modifying drive related factors is another option. ' $\mathrm{GH}$ ' describes his approach, "I look at the travel time. What about traffic at this time of day?' And 'RL' explains that drive time estimates can be significantly different for the same distance depending on factors such as 'is the weather inclement or clear?' Some of this decision-making can be assisted by general planning on the part of paramedics. As 'GH' explains, 'For driving - you really need to know your area'. This knowledge helps fill in the blanks when estimating drive times and considering route alternatives. 


\section{Discussion}

Operationally speaking, ambulance service referral of emergency patients directly to regional medical facilities on a widespread basis is a relatively new development for EMS. The historic mandate of land ambulance services involves providing a uniform system of transporting patients directly to a local medical facility, with minimal attention required or given to consideration of alternative destinations. This new, regionalised transport procedure is more complicated than just transportation to a condition-specific facility. Consistent with referrals within the medical community it involves a high stakes request for specialised assistance from one provider (paramedic) and acceptance by another (physician).

Referral program standards require that the selection of patients be based on explicit clinical criteria developed by specialists including emergency physicians, neurologists and interventional cardiologists. These criteria however, are decontextualised in the sense that they list inclusion and exclusion criteria, special considerations, indications, contraindications and so on, which paramedics are then required to apply to the context of a specific patient.

Such a major change in practice introduces new ethical considerations with major implications for clinical decision making and obliges paramedics to at least tacitly re-visit their first principles of always transporting patients to the closest hospital emergency department; and making destination decisions independent of other health care professionals. In response, paramedics describe the establishment of a unique ethical approach that is both customised to the pre-hospital referral context and consistent with aspects of professionalism. Demonstrating professionalism by managing complex ethical dilemmas is frequently cited as an essential element of a profession's responsibilities and paramedics' experiences in this regard are discussed in the following sections.

When Freidson (22) discusses the concept of professional ethicality he describes a 'set of attitudes held by individuals' ( $p$. 378). These stand in contrast to specific concrete skills for which expertise can be demonstrated. Rather, these are the subjective views of a practitioner that can guide use of these skills. During referrals, paramedics describe their heavy reliance on the application of beneficence as a motivator. This beneficence is informed by paramedics' awareness of the vulnerability of their patients in terms of minimal control over their acute situation. For example, ambulance patients have no choice with respect to which or when paramedics provide their pre-hospital care. They are also often experiencing unique, frightening and high-stress situations, which contributes to an overall context of vulnerability.

Of course there are other ethical principles that come into play during referrals. The complex situations within which referrals occur involve weighing other principles such as patient autonomy (related to consent), veracity (by providing truth to patients and colleagues - such as those accepting the referral), and avoidance of malfeasance (causing harm associated with transport risks). This involves more than technical competence in terms of assessing a patient as meeting clinical criteria for referral. It involves a level of complexity that is entirely consistent with that required of professionals, as outlined in Purtilo and Doherty's (11) discussion about the ethical characteristics of a caring professional.

In the field paramedics are often faced with uncertainty as they are dealing with real patients who may benefit from a procedure, but for whom the information available is often vague. When this uncertainty creates cases for which eligibility for referral is borderline, paramedics actively advocate. For example, paramedics are very aware of the unreliability of descriptions of time that patients and their families provide during a crisis, particularly when the unit of measure must be in minutes. Consequently, the context of the call requires paramedics to construct a timeline with varying amounts of precision. For paramedics, their belief that they have a duty to advocate for these patients is similar to that of other health care providers who demonstrate their professionalism by enshrining this type of duty in codes of professional conduct $(23,24)$.

It is important to remember that this advocacy takes place in a time-limited context, and this may reinforce the authority of paramedics given that patients are aware the paramedics have experience with these situations. Patients are inclined to defer to the more knowledgeable paramedic due in part to their status as a formal health care provider essentially establishing a situation of acute-trust. In responding to this trust paramedics provide a voice for patients' interests (23) and engage in inter-professional collaboration in an effort to tip the scales of referral in the patients favour.

In doing so paramedics are aware that they also have a commitment to the clinical criteria for referrals, and by extension the specialists who ultimately decide on a course of treatment. This creates a type of dual allegiance (24) and so paramedics are careful to only advocate in cases they judge to have clear merit. Nevertheless, paramedics must navigate ethical tensions. For example, when augmenting their advocacy, paramedics encounter a 'push-pull' tension created between driving quickly motivated by beneficence, and risks of malfeasance (potential harm) associated with increased speed.

This type of complex situation is not unlike that experienced by other health professionals when exposing themselves to review by colleagues. Paramedics accept these risks, which are unavoidable in a system of professions, where the visibility of one's performance to colleagues or superiors is necessarily fragmentary or partial (22). This is further illustrated by another example: only the paramedics know what inter-personal dynamics occurred at the scene during a referral decision, yet the evaluation of the decision is post-hoc at the regional centre. In sum, this unique form of advocacy is consistent with Hanks (23) view that professional advocacy for patients can take many forms, as influenced by the context of practice. 


\section{Conflict of interest}

The author has completed the ICMJE conflict of interest statement. The author declares he has no competing interests.

\section{Conclusion}

Paramedics encounter ethical dilemmas as a consequence of their responsibilities during EMS referrals and have responded by developing a type of ethicality that is consistent with professionalism in ethical practice as described in the literature. This professionalism is customised to the unique character of their field of practice and includes applying beneficence, working in ethically complex contexts and advocating for patients.

\section{Limitations}

This study is admittedly exploratory, and this area of research would benefit from more focused inquiry into paramedical ethics. Consistent with the basic assumptions of qualitative inquiry, this study provides in-depth attention on context and meanings held by participants. Readers are reminded however, that interview based studies are not observational studies and readers must make their own decisions about the validity of findings in relation to their own knowledge.

Finally, inquiries into non-technical aspects of paramedicine are few and far between, yet they offer important insights into the true nature of paramedicine. Other inquiries should be developed to assist in this regard.

\section{References}

1. Freidson E. Professionalism: The third logic. Chicago: University of Chicago Press; 2001.

2. Ontario Ministry of Health and Long-Term Care, Basic Life Support Standards Patient Care Standards; 2007. Available at: www.health.gov.on.ca/english/public\%5Cprogram/ehs/edu/ pdf/bls_patient.pdf

3. Paramedic Association of Canada; 2015. Available at: www. paramedic.cal

4. Shields A, Flin R. Paramedics' non-technical skills: a literature review. Emerg Med J 2013;30:350-4.

5. Trivendi K, Jeremiah DS, Cone DC. Can paramedics read ST-segment elevation myocardial infarction on prehospital 12lead electrocardiograms? Prehosp Emerg Care 2009;13:20714.

6. Chenkin J, Gladstone D J, Verbeek PR, et al. Predictive value of the Ontario Prehospital Stroke Screening Tool for the identification of patients with acute stroke. ibid. 2009;13:1539.
7. Lee $\mathrm{CH}$, Van Gelder CM. Cone, DC. Early cardiac catheterization laboratory activation by paramedics for patients with ST-segment elevation myocardial infarction on prehospital 12-lead electrocardiograms. ibid. 2010;14:153-8.

8. Tai YJ, Yan B. Minimising time to treatment: targeted strategies to minimise time to thrombolysis for acute ischaemic stroke. Intern Med J 2013;43:1176-82.

9. Bloom BS, editor. Taxonomy of Educational Objectives. Vol. 1: Cognitive Domain. New York: McKay, 1956.

10. Jensen J. Paramedic clinical decision-making: results of two Canadian studies. International Journal of Paramedic Practice 2011;1:186-93.

11. Purtilo RB, Doherty RF. Ethical dimensions in the health professions. St. Louis, MO: Elsevier Saunders, 2011.

12. Bigham B, Blanchard I, Jensen J, et al. Ethical dilemmas: an intro to ethics in EMS research. Canadian Paramedicine. Available at: http://emsnews.com/features/spotlight-onscience/ethical-dilemmas

13. Jensen J, Croskerry P, Travers AH. Paramedic clinical decision-making during high acuity emergency calls: design and methodology of a Delhi study. BMC Emerg Med 2009;9:1-4.

14. Metz D. Running hot: structure and stress in ambulance work. Cambridge, MA: Abt Books; 1981.

15. Nelson BJ. Work as a moral act: how emergency medical technicians understand their work. In: Barely S and Orr J, editors. Between Craft and Science, Ithaca: Cornell University Press; 1997. p. 154-84.

16. Campeau A. The space control theory of paramedic scene management. Symbolic Interaction 2008;31:285-302.

17. Campeau A. The paramedic kairotope theory: Findings. Journal of Paramedic Practice 2011;3:376-80.

18. Guba EG, Lincoln YS. Competing paradigms in qualitative research. In: Denzin NK and Lincoln YS, editors. Handbook of Qualitative Research. Thousand Oaks, CA: Sage; 1994. p. 105-17.

19. Stauss A, Corbin J. Basics of qualitative research: techniques and procedures for developing grounded theory. 2nd edn. Thousand Oaks, CA: Sage; 1999.

20. Charmaz K. Constructing grounded theory: a practical guide through qualitative analysis. Thousand Oaks, CA: Sage; 2006.

21. DiCicco-Bloom B, Crabtree BF. Making sense of qualitative research: the qualitative research interview. Med Educ 2006;40:314-21.

22. Freidson E. Profession of medicine: a study of the sociology of applied knowledge. Chicago, IL: University of Chicago Press; 1988.

23. Hanks RG. The medical-surgical nurse perspective of advocate role. Nursing Forum 2010;45:97-107.

24. Trapani J. Critical care nurses as dual agents: enhancing inter-professional collaboration or hindering patient advocacy? Nurs Crit Care 2014;19:219-21. 